\title{
Correlatos de la terapia EMDR en la neuroimagen funcional y estructural: Un resumen crítico de los hallazgos recientes
}

\author{
Marco Pagani \\ Instituto de Ciencias y Tecnologías Cognitivas, CNR, Roma, Italia \\ Göran Högberg \\ Departamento de Neurociencia Clínica, Sección de Psiquiatría, Huddinge, Instituto Karolinska, Estocolmo, Suecia \\ Isabel Fernandez \\ Asociación EMDR Italia, Bovisio Masciago (MI), Italia \\ Alberto Siracusano \\ Departamento de Medicina de Sistemas, Universidad de Roae "Tor Vergata," Roma, Italia
}

\begin{abstract}
Las investigaciones con neuroimagen sobre los efectos de las psicoterapias que tratan el trastorno de estrés postraumático (TEPT), incluyendo la terapia de desensibilización y reprocesamiento por movimientos oculares (EMDR), han mostrado hallazgos conformes con modificaciones en el flujo sanguíneo cerebral (FSC; tomografía computerizada de emisión monofotónica [SPECT]), en el volumen y la densidad neuronal (imágenes por resonancia magnética [MRI]) y, más recientemente, en las señales eléctricas del cerebro (electroencefalografía [EEG]). Además, en el pasado reciente, se han monitorizado, por medio de EEG, cambios neurobiológicos relacionados con EMDR durante la misma terapia y han mostrado un cambio en la activación máxima desde las regiones límbicas emocionales a las regiones cognitivas corticales del cerebro. Esta ha sido la primera vez que se han señalado los cambios neurobiológicos que tienen lugar durante una sesión de psicoterapia, lo que ha hecho que EMDR sea la primera psicoterapia con un efecto neurobiológico probado. El propósito de este artículo ha sido analizar los resultados de los cambios funcionales y estructurales que han tenido lugar durante el tratamiento del TEPT y que han sido presentados por diversos grupos de investigación durante el período entre 1999 y 2012. Los cambios fisiopatológicos referidos se presentan en función de las técnicas neuropsicológicas y la metodología implementadas y son analizados de manera crítica.
\end{abstract}

Palabras clave: EMDR; neurobiología; EEG; SPECT; MRI; sistema límbico

$\mathbf{L}$ as técnicas no invasivas de grabación de la actividad cerebral se clasifican en dos amplias categorías. Las primeras son las técnicas electromagnéticas: electroencefalografía (EEG), potenciales relacionados con eventos (PRE) y magnetoencefalografía. Estas tienen una resolución temporal excelente (unos pocos milisegundos), pero se combina con una resolución espacial bastante mala (varios centímetros). Las segundas son las técnicas relacionadas con la hemodinámica y el metabolismo: imagen por resonancia magnética funcional (fMRI), tomografía computerizada de emisión monofotónica (SPECT), tomografía por emisión de positrones (PET) y espectroscopia por luz casi infrarroja (NIRS). Estas miden el flujo sanguíneo cerebral regional (FSCr) y los cambios metabólicos como indicadores de la actividad neural y son adecuadas para investigar de manera fiable las áreas profundas del cerebro. Además, al contrario que las técnicas electromagnéticas, estas últimas tienen una buena resolución espacial $(1-6 \mathrm{~mm})$ pero mala

This article originally appeared as Pagani, M., Högberg, G., Fernandez, I., \& Siracusano, A. (2013). Correlates of EMDR Therapy in Functional and Structural Neuroimaging: A Critical Summary of Recent Findings. Journal of EMDR Practice and Research, 7(1), 29-38. Translated by Miriam Ramos Morrison. 
resolución temporal (varios segundos para la fMRI y del orden de unos minutos para la PET/CT; Plailly, Demarquay y Royet, 2009).

La baja resolución temporal es una clara desventaja para las técnicas de PET/CT y fMRI al compararlas con la de las técnicas electromagnéticas, que pueden monitorizar la actividad neural e identificar cambios que están ocurriendo casi en tiempo real. Por otro lado, la mejor resolución espacial de las técnicas de PET/CT y fMRI hace que sean particularmente útiles a la hora de revelar tanto pequeños clusters de activación como redes distribuidas a gran escala, incluyendo estructuras cerebrales tanto corticales como profundas (Cabeza y Nyberg, 2000).

\section{Neuroimagen y el trastorno de estrés postraumático}

Se han utilizado técnicas no invasivas de grabación de la actividad cerebral para estudiar los cambios anatómicos y funcionales en individuos con trastorno de estrés postraumático (TEPT). Estudios funcionales (SPECT, PET y fMRI) y estructurales (MRI) han mostrado alteraciones neurobiológicas significativas en pacientes con TEPT durante la exposición a una visualización autobiográfica del trauma dirigida por una narrativa o script (Bremner, 2007). Estos estudios han dado lugar a la identificación de cambios metabólicos y morfológicos en el cerebro, que ocurren cuando la enfermedad se vuelve sintomática, y han aportado información sobre el rol de cada área específica en los complejos mecanismos implicados en el procesamiento de las emociones y el trauma psicológico. (Para un análisis extenso, véase Bremner, 2007.)

En el caso del TEPT, cambios (tanto aumentos como disminuciones) en el riego sanguíneo, el metabolismo y el volumen de materia gris han sido encontrados en el hipocampo, la amígdala, el córtex prefrontal medial, el cíngulo anterior y posterior y el córtex temporal (véase una revisión de Francati, Vermetten y Bremner, 2007). Todas estas regiones pertenecen al sistema límbico que se encuentra particularmente implicado en el procesamiento de las emociones tanto positivas como negativas. Cuando se combinan los resultados de estos estudios, inequívocamente muestran en los pacientes, después de un evento traumático, un procesamiento patológico de los recuerdos traumáticos asociado con una correspondiente reducción del control del córtex prefrontal medial y el cíngulo anterior sobre la amígdala hiperreactiva, que se piensa son los mecanismos funcionales centrales implicados en el TEPT.
El propósito de este artículo es doble: (a) Presentar y discutir brevemente los artículos disponibles (indexados en PubMed 1999-2012) en los que los efectos neurobiológicos de diversas psicoterapias hayan sido investigados por técnicas de neuroimagen y (b) centrarse en los avances de la investigación con neuroimagen con la terapia de sensibilización y reprocesamiento por movimientos oculares (EMDR) y presentar de manera crítica los resultados más recientes.

\section{Neuroimagen en las psicoterapias para el trastorno de estrés postraumático}

Las técnicas de neuroimagen han sido utilizadas en un intento de aclarar los correlatos funcionales y anatómicos de diversas psicoterapias y revelar sus efectos neurobiológicos. En un extenso análisis, Roffman, Marci, Glick, Dougherty y Rauch (2005) analizaron 14 investigaciones funcionales de neuroimagen en las que se medían los efectos de diversas psicoterapias sobre la función cerebral. Pese a los resultados clínicos positivos y los razonables efectos de las terapias conductuales, cognitivo conductuales e interpersonales sobre las funciones cerebrales, los cambios neuroanatómicos fueron inconsistentes, tanto entre trastornos como entre psicoterapias, lo cual impidió alcanzar una conclusión bien estructurada. Sin embargo, los estudios analizados se llevaron acabo con diversas metodologías, paradigmas experimentales y psicoterapias, pero lo que es más importante, con grupos de pacientes que pertenecían al espectro completo de diagnósticos psiquiátricos, desde la depresión mayor hasta las fobias y la esquizofrenia. Dicha heterogeneidad dio lugar a dificultades a la hora de sugerir mecanismos fisiológicos plausibles y convergentes para los tratamientos investigados. Para evitar esto, en este análisis tan sólo se incluyen artículos en los cuales la psicoterapia fue utilizada para tratar el TEPT. Analizaremos dichos estudios separando las diferentes técnicas y psicoterapias.

\section{Imagen por resonancia magnética}

Centrándose en el TEPT, en 2005, Lindauer et al. señalaron el efecto de la psicoterapia breve ecléctica (PBE) sobre el volumen del hipocampo. Este estudio de MRI de alta definición mostró en su línea basal un hipocampo generalmente más pequeño en 18 pacientes en comparación con 14 controles traumatizados. En los 9 pacientes asignados al grupo de tratamiento, dicho volumen no fue modificado por la PBE en las estructuras investigadas segmentadas de manera manual (hipocampo, amígdala y giro parahipocampal) pese al efecto positivo de la psicoterapia sobre los síntomas 
del TEPT (Lindauer et al., 2005). Sin embargo se encontró que los hipocampos eran más pequeños en los pacientes que en los controles traumatizados - un resultado que se reproduce a menudo en la investigación del TEPT. La cuestión de si esta condición anatómica es una característica de rasgo (presente antes de los traumas principales) o de estado (posterior al trauma principal) aún no ha sido esclarecida. Además, dada la falta de seguimiento de los pacientes, el estudio no aclaró de manera concluyente los efectos de la terapia sobre las estructuras subcorticales. De hecho, el tiempo relativamente corto de la terapia (4 meses) y el tiempo mínimo general transcurrido entre el final de la psicoterapia y el MRI pueden no haber sido suficientemente largos como para producir cambios anatómicos detectables, que posiblemente tengan lugar tras un periodo más largo después del éxito del tratamiento.

\section{Tomografía computerizada de emisión monofotónica}

Durante los años siguientes, el mismo grupo, siempre utilizando la PBE, investigó el flujo sanguíneo cerebral (FSC) en 10 participantes por medio de la SPECT y señaló que después de recibir psicoterapia con resultado clínico positivo, la activación encontrada durante la escucha del script en la línea basal disminuía de manera significativa en el giro frontal medio. Es más, la eficacia del tratamiento, medida por las puntuaciones de TEPT, se correlacionaba de manera positiva con el FSC en el córtex temporal y frontal (Lindauer et al., 2008). Sin embargo, este estudio fue realizado con una cámara SPECT de baja resolución y el umbral de diferencias estadísticas fue establecido a un nivel liberal de $\mathrm{p}<.01$ no corregido para múltiples comparaciones a nivel vóxel.

Previamente, Peres et al. (2007) habían publicado un estudio con 16 pacientes con TEPT antes y después de asistir a terapia de reestructuración cognitiva. La investigación fue realizada utilizando un umbral estadístico bajo $(\mathrm{p}<.001$ no corregido para múltiples comparaciones) y refería, después de una psicoterapia con éxito, una activación más alta en las regiones cortical (lóbulos temporal, parietal y prefrontal) y subcortical (tálamo) en el hemisferio izquierdo durante un paradigma provocador dirigido por un script.

\section{Neuroimagen en EMDR}

La terapia EMDR (Shapiro, 2001) ha sido reconocida como un método basado en la evidencia para el tratamiento del trauma psicológico y otros trastornos de ansiedad (Ehlers et al., 2010), aunque la ma- nera en que actúa sobre los circuitos neurales está lejos de estar totalmente aclarada. Un mecanismo putativo detrás de la eficacia del EMDR postula la hipótesis de que los recuerdos patológicos son retenidos en las sinapsis de la amígdala después de una sobrepotenciación de los receptores del ácido alfaamino-3hidroxi-5-metil-4-isoxazol (AMPA). Dicho fortalecimiento podría impedir que los recuerdos mórbidos se uniesen por medio de la corteza del cíngulo anterior al rastro del recuerdo cognitivo y fuesen almacenados adecuadamente (para una revisión, véase Harper, Rasolkhani-Kaòhorn y Drozd, 2009). En animales, la estimulación tetánica de baja frecuencia (de 1 a 5 pulsaciones por segundo [hertz]) causó en la amígdala una despotenciación de los receptores del AMPA proporcional a la frecuencia de estimulación, borrando por tanto los recuerdos traumáticos (Bender, Bender, Brasier y Feldman, 2006; Hölscher, Anwyl y Rowan, 1997). Dicha excitación neuronal se parece a la que producen los terapeutas durante una sesión de EMDR (unos $2 \mathrm{~Hz}$ ) y el sutil mecanismo neurobiológico positivo de la terapia puede estar relacionado con una tasa reducida de despolarización de las sinapsis del sistema límbico provocado por la estimulación sensorial bilateral. Los recuerdos emocionales conservados de manera patológica en la amígdala se desplazarán entonces al córtex cerebral y serán elaborados adecuadamente (Lin, Yeh, Lu y Gean, 2003). A nivel macroscópico, los recientes hallazgos de hiperactivación del giro parahipocampal y las cortezas límbicas durante la terapia EMDR (Pagani et al., 2012) parecen apoyar dicha hipótesis y animan a que se realicen más investigaciones sobre este fascinante tema.

Se ha realizado todo un cuerpo de investigación para evaluar la efectividad del EMDR y gran cantidad de estudios están centrados en investigar su sustrato neurobiológico a pesar de las dificultades que surgen de la resolución tanto temporal como espacial de las técnicas seleccionadas. La base neurobiológica de la efectividad del EMDR en el tratamiento del TEPT había sido apoyada por estudios de SPECT, que después de comparar la actividad cerebral antes y después de la terapia han demostrado que ocurren cambios significativos en el flujo sanguíneo, principalmente en las áreas límbicas y el córtex prefrontal. En general, los resultados de estos estudios indican que después del tratamiento hay una inversión de las anormalidades prefrontales y límbicas, que fueron claramente reconocidas al inicio del tratamiento y son un hallazgo frecuente de los estudios de neuroimagen en pacientes con TEPT. De hecho, a pesar de la advertencia de la relativamente baja resolución espacial del SPECT, 
el aumento del flujo sanguíneo después del tratamiento en el córtex frontal lateral y medio puede reflejar un mayor control sobre la amígdala y un aumento de la estabilización de la hiperactivación cerebral patológica, dando lugar a una reducción de los síntomas somatosensoriales de ansiedad. Estos resultados son consistentes con mejorías clínicas, incluyendo depresión y trastornos afectivos en general, lo que demuestra que EMDR tiene un impacto significativo sobre las funciones cerebrales y que el patrón de actividad cerebral emergente post-EMDR es consistente con los cambios que pueden estar mitigando las condiciones postraumáticas y de ansiedad.

\section{Tomografía computerizada de emisión monofotónica}

El primer estudio en el que se utilizó la SPECT en investigaciones de EMDR data de 1999, cuando Levin et al. publicaron un informe de un caso sobre un participante con TEPT. Al recordar el evento traumático, la SPECT mostró un aumento del FSC después de la terapia en el cíngulo anterior y el lóbulo frontal izquierdo. Desafortunadamente, los autores, a pesar de analizar extensamente los resultados clínicos y neuropsicológicos positivos después de la terapia EMDR, tan solo mencionaron los efectos funcionales y el diseño del estudio SPECT (comparativa de un solo participante). De hecho, los resultados del análisis SPECT omiten información sobre el tipo de cámara y su resolución y no ofrecen ningún detalle sobre la metodología utilizada para el análisis de las imágenes, ni para los niveles estadísticos, si es que los hay. Sin embargo, a pesar de las limitaciones mencionadas, el estudio abrió el camino para los siguientes que demostraron la viabilidad de investigar la patofisiología cerebral durante la re-experimentación del trauma

En 2005, Lansing, Amen, Hanks y Rudy investigaron la activación cerebral durante el recuerdo de un evento traumático en seis policías traumatizados psicológicamente antes y después de la terapia EMDR. Después de que hubieran desaparecido los síntomas clínicos y neuropsicológicos del TEPT, encontraron un descenso importante del flujo cerebral en los lóbulos occipitales, el lóbulo parietal izquierdo y los lóbulos frontales posteriores, así como un aumento significativo de la perfusión en el giro frontal inferior izquierdo. El estudio fue realizado con una cámara SPECT de alta resolución y con un umbral estadístico aceptable, considerando el bajo número de participantes y la naturaleza exploratoria de la investigación. Los resultados más relevantes después de EMDR fueron el descenso paralelo de la perfusión en las regiones hiperactivadas durante la fase sintomática y el aumento del flujo sanguíneo en el córtex frontal inferior. Éstos hallazgos indirectamente confirman, a nivel funcional, el impacto de EMDR sobre la neurobiología del TEPT al revertir el reducido control del córtex prefrontal sobre la amígdala.

Considerando los escasos informes sobre un tema potencialmente tan interesante-los cambios que ocurren en el cerebro con las psicoterapias en general y con la eliminación de los síntomas relacionadosnuestro equipo de investigación se ha comprometido a contribuir investigando los eventos neurobiológicos que ocurren a nivel anatómico y funcional con la terapia EMDR. Los estudios realizados de esta manera fueron parte de un amplio proyecto de investigación sobre TEPT entre empleados del transporte público de Estocolmo que habían experimentado bien un atropello a una persona con el tren o bien un atraco en el trabajo (Högberg et al., 2007, 2008 [estudios clínicos]; Looi et al., 2008, 2009; Nardo et al., 2010 [estudios de MRI]; Pagani et al., 2005; Pagani et al, 2007; Nardo et al., 2011 [estudios de SPECT]). En todas estas investigaciones, la entrevista clínica estructurada para los trastornos del eje 1 del Manual diagnóstico y estadístico de los trastornos mentales, $4^{a}$ edición (DSM-IV) (SCID-I; First, Gibbon, Spitzer y Williams, 1997) sentó las bases para el diagnóstico clínico. Es más, los resultados de las escalas basadas en entrevistas y las escalas de auto-evaluación constituyeron las bases de la evaluación neuropsicológica.

El proyecto fue inspirado por un meta-análisis de 61 estudios de resultados sobre el TEPT, en los cuales los pacientes fueron tratados con terapias farmacológicas y psicológicas tales como terapia conductual, EMDR, entrenamiento en relajación, hipnoterapia y terapia dinámica (Van Etten y Taylor, 1998). El estudio concluyó que las mejores terapias psicológicas eran la terapia cognitivo conductual (TCC) y el EMDR y que estas psicoterapias eran más efectivas que la terapia farmacológica. El 32\% de los pacientes medicados dejaron el tratamiento frente al $14 \%$ de los pacientes tratados con terapias psicológicas. Otro meta-análisis llegó a la conclusión de que el EMDR y las terapias de exposición tenían resultados clínicos positivos en el tratamiento del TEPT (Davidson y Parker, 2001); y Bradley, Green, Russ, Dutra y Westen (2005) indicaron que en más de la mitad de los pacientes que completaron el tratamiento de TCC o EMDR, los síntomas generales mejoraron. Sin embargo, en todos estos estudios, los pacientes fueron monitorizados durante menos de 12 meses y Bradley et al. señalaron la falta de seguimiento a largo plazo. Esta advertencia fue superada por Högberg et al. (2008) quienes indicaron 
un resultado positivo de la terapia EMDR tres años después de la última sesión.

Los resultados preliminares de Lansing et al. (2005) fueron confirmados en 2007 por Pagani et al. (2007) en el estudio SPECT más amplio que hasta la fecha haya investigado los cambios en el FSC que tienen lugar después de la terapia EMDR. Quince pacientes fueron escaneados antes y después de la intervención terapéutica. Para aumentar la fiabilidad del estudio y la robustez de los resultados, un grupo control de 22 participantes no sintomáticos que habían sufrido el mismo trauma que los pacientes fue incluido en el estudio. Esta última precaución metodológica es de la mayor relevancia porque minimiza el posible sesgo en los resultados debido a la heterogeneidad psicológica entre los dos grupos. Es más, se aplicó un umbral estadístico muy estricto (corrección de la falsa tasa de descubrimiento tanto a nivel de vóxel como de cluster) aceptando el riesgo de falsos negativos debido a errores estadísticos del tipo 2. Cuando se comparó a los 11 participantes del tratamiento con los controles, las significativas diferencias de grupo encontradas antes de la terapia EMDR desaparecieron después del tratamiento. Es más, los participantes mostraron, después de la terapia, una importante normalización del FSC en el córtex visual parieto-occipital y en el hipocampo y un aumento en el córtex prefrontal lateral.

Tomados en su conjunto, los resultados de los últimos dos estudios indican que el descenso del flujo cerebral regional después del éxito de la terapia EMDR se asoció con la remisión de síntomas como flashbacks, recuerdos estresantes intrusivos, alucinaciones y re-experimentación persistente del trauma a nivel somático. Por otro lado, EMRD normalizó la capacidad de recuperar aspectos importantes del trauma y mejoró la atención y el sentido del yo. Además, la activación del córtex prefrontal, asignado a la inhibición del sistema límbico en respuesta a los estímulos patológicos que se parecen al evento traumático, recuperó su rol inhibidor reduciendo la hiperactivación de la amígdala y la hiperactivación cortical relativa.

El estudio SPECT EMDR más reciente hasta la fecha fue realizado por Oh y Choi (2007) en dos pacientes que sufrían de trauma psicológico relacionado con un accidente de tráfico. Los autores encontraron, después de EMDR, un aumento de la perfusión cerebral en el córtex prefrontal dorsolateral bilateral y un descenso de la misma en el córtex asociativo temporal. Además, las imágenes SPECT se compararon con aquellas de un grupo control no traumatizado y los resultados estaban en consonancia con los anteriores, lo que indica una normalización de la absorción de marcadores después de la terapia EMDR. En cuanto al primer estudio de Levin, la importancia de los resultados de esta investigación se ve afectada por la cantidad tan baja de pacientes en las muestras, así como por la relativa pobreza de los datos estadísticos $(\mathrm{p}<.01)$. Sin embargo, el estudio también confirmó el efecto neurobiológico general de EMDR, con una tendencia a la restauración del control cortical sobre las hiperactivadas estructuras clínicas subcorticales.

Imagen por resonancia magnética e imagen por resonancia magnética funcional

Más recientemente, algunos estudios que han aplicado MRI y fMRI también han tratado de dar una idea de la neurobiología del EMDR. En 2007, Letizia et al. informaron de un caso de un paciente con TEPT en el que se delinearon manualmente los hipocampos en imágenes por resonancia magnética de alta resolución. Después de 8 semanas de tratamiento EMDR, el paciente ya no cumplía con los criterios de TEPT según la evaluación clínica y mostraba un aumento bilateral de los volúmenes del hipocampo. Los autores concluyeron que los cambios volumétricos ocurridos en un período de tiempo relativamente tan corto podrían haber sido causados bien por la neurogénesis o por el aumento del contenido de agua y electrolitos en los hipocampos, pero dejaron la puerta abierta a la hipótesis de que tanto la psicoterapia como la farmacoterapia podrían inducir alteraciones en la expresión genética y cambios estructurales en el cerebro. El mismo grupo ha realizado recientemente, con la misma metodología, un segundo estudio (Bossini et al., 2012) con el objetivo de investigar el volumen del hipocampo en 10 pacientes con TEPT tratados con EMDR. Encontraron que después de la terapia, el volumen del hipocampo creció un $6 \%$ de nuevo en asociación con una mejoría en los síntomas de TEPT. En este artículo, los autores no hicieron especulación alguna sobre la naturaleza de este fenómeno, ya que creen que son necesarios más estudios en un futuro, posiblemente usando una cohorte más amplia de participantes traumatizados, así como comparando los datos estructurales con los de un grupo control, para investigar los supuestos mecanismos funcionales que subyacen a estos cambios anatómicos.

Un estudio de resonancia magnética estructural, que incluyó el mismo grupo de pacientes que en Pagani et al. (2007), demostró una disminución de la densidad de la materia gris en varias regiones límbicas en los pacientes que no respondieron a la terapia con EMDR en comparación con aquellos para los que el tratamiento resultó en la desaparición de los síntomas (Nardo et al., 2010). En las cortezas parahipocampal, insular 
y del cíngulo posterior, se encontró que menores concentraciones de materia gris estaban relacionadas con terapias de TEPT y EMDR. Se sabe que estas regiones están implicadas en procesos tales como la integración, codificación y recuperación de recuerdos autobiográficos y episódicos; el procesamiento emocional (esto es, condicionamiento clásico, valoración cognitiva, experiencia de estados de sensación); la conciencia interoceptiva; y la experiencia autorreferencial consciente. La capacidad de respuesta al EMDR, que en parte tiene como objetivo mejorar tal sintomatología, se correlacionó de manera preliminar con los mismos sustratos estructurales. La importancia de este estudio, galardonado con el Premio Francine Shapiro en 2011 por la Asociación Europea de EMDR, radica en el hecho de que la resonancia magnética estructural se realizó sólo una vez, por lo que era una representación estática de la neurobiología del cerebro antes de la terapia EMDR. Los pacientes que no respondieron a la terapia fueron aquellos en los que la densidad de materia gris se encontraba más dañada y se pensó que esto podría haber interferido con la eficacia de la terapia EMDR. Así como en el estudio de Bossini et al. (2012), son necesarios grupos más grandes de pacientes para determinar si este hallazgo está relacionado con un factor de rasgo o si dicha disminución de la densidad neuronal estaba causada por el impacto del trauma en la neurobiología de los pacientes.

En 2009, Richardson et al. grabaron la señal de oxigenación de la sangre durante una sesión de EMDR auto-administrada por un terapeuta en el gantry de la cámara magnética y encontraron, en comparación con el estado de reposo, un marcado cambio en la activación cerebral en la corteza prefrontal, lo que demostraba un cambio ventromedial que se extendía a varias regiones límbicas. Especularon que el patrón de resultados fue consistente con la hipótesis de que el protocolo EMDR (en particular, la estimulación bilateral alterna) recluta una red cortical grande para mejorar la gravedad de los síntomas del TEPT. Sin embargo, más allá de ser un caso clínico, el estudio funcional se realizó en un entorno de análisis no ecológico, frío y antinatural, lo que dio lugar a posibles sesgos causados por el malestar físico y psicológico de los pacientes durante las condiciones experimentales (Mazard et al., 2002).

\section{Espectroscopia de luz casi infrarroja}

Ohtani, Matsuo, Kasai, Kato y Kato (2009) utilizando la espectroscopia de luz casi infrarroja (NIRS, por sus siglas en inglés) realizaron un estudio pionero monitorizando directamente los cambios hemodinámicos del cerebro durante el proceso de reducir la angustia del recuerdo relacionado con el trauma por medio de EMDR. Utilizando tareas basadas en los procedimientos de EMDR, también examinaron los cambios longitudinales relacionados con el tratamiento. Aunque la investigación se confinó al córtex frontal, dado el limitado potencial de la técnica, se encontró que recordar a la vez que se hacen movimientos oculares se asocia con un descenso importante en la concentración de hemoglobina oxigenada en el córtex prefrontal lateral en comparación con el recuerdo sin movimientos oculares. Longitudinalmente, la concentración de hemoglobina oxigenada durante la evocación del recuerdo disminuía de manera significativa y esta reducción se correlacionaba con una mejoría clínica cuando los datos posteriores al tratamiento se comparaban con los datos previos al tratamiento. No era probable que los resultados fueran atribuidos a cambios en el sistema circulatorio global causados por la activación autonómica porque no hubo alteración del ritmo cardíaco durante el recuerdo del trauma. Los autores sugirieron que la eficacia de EMDR en pacientes con TEPT podría estar asociada con la reducción de la sobreactivación del córtex prefrontal lateral durante la evocación del recuerdo relacionado con el trauma con movimientos oculares. Sin embargo, la técnica NIRS limita el análisis funcional a un volumen fino de tejidos justo debajo de la superficie del cráneo y la mayoría de los sistemas incluyen pocos detectores que cubren tan sólo una pequeña parte del córtex cerebral, lo que impide un análisis de todo el cerebro.

\section{Electroencefalografía}

También se realizaron esfuerzos para descubrir el impacto del componente de la estimulación bilateral de la terapia EMDR. En 2004, Lamprecht et al. investigaron con EEG los potenciales cerebrales relacionados con el evento (PRE) antes y después de una sesión de tratamiento con EMDR. El resultado más interesante se obtuvo para el PRE P3a (un componente de señales de interrupción), conocido por su asociación con la implicación de la atención y la evaluación de eventos nuevos para preparar la acción conductual posterior. Tan sólo el grupo de pacientes mostró una mejoría significativa del PRE P3a en la sesión posterior al tratamiento, sugiriendo que la mejoría clínica puede estar relacionada con cambios en el procesamiento de información asociados a una orientación reducida a estímulos nuevos y a un nivel reducido de activación después del tratamiento.

Harper et al. (2009) Informaron que las grabaciones de EEG de sesiones de EMDR mostraron que una baja estimulación del cerebro invoca una respuesta más amplia de las áreas de la memoria. Esta respuesta 
está en una frecuencia de unos $1.5 \mathrm{~Hz}$, lo que demuestra que la frecuencia y el poder de las ondas inducidas por la estimulación bilateral del cerebro durante la terapia EMDR son similares a aquellas de las oscilaciones lentas y las ondas delta del sueño de ondas lentas, particularmente en las regiones frontopolares. Especularon que la despotenciación de las sinapsis del recuerdo del miedo en la amígdala altera los circuitos del recuerdo del miedo. Ese cambio mecánico resulta en un cambio perceptivo beneficioso. También fue significativo ver que el estado cerebral establecido durante EMDR era similar al del sistema de edición de memoria que ocurre durante el sueño de ondas lentas.

De modo similar, Propper y colaboradores (Propper y Christman, 2008; Propper, Pierce, Geisler, Christman y Bellorado, 2007) examinaron en participantes normales el efecto de los movimientos oculares utilizados en EMDR en la coherencia EEG interhemisférica. Encontraron una coherencia reducida de la frecuencia gamma asociada con 30 segundos de movimientos oculares bilaterales con los ojos abiertos. Se vio que la manipulación de movimientos oculares utilizada en este estudio facilitaba la memoria episódica y resultaba en un descenso de la coherencia EEG interhemisférica en el córtex prefrontal anterior. La conclusión fue que al cambiar la coherencia interhemisférica en las áreas frontales, los movimientos oculares utilizados en EMDR fomentan la consolidación de los recuerdos traumáticos, reduciendo por tanto los recuerdos intrusivos que encontramos en este trastorno.

Por otro lado, la hipótesis de interacción interhemisférica en la terapia EMDR fue cuestionada por Samara, Elzinga, Slagter y Nieuwenhuis (2011), quienes mostraron que los participantes sanos recordaban más palabras emocionales (pero no neutrales) después del procedimiento de movimientos oculares que después del procedimiento de control. Sin embargo, en su experimento, el análisis de EEG no indicó evidencia alguna de que los movimientos oculares alterasen la coherencia interhemisférica de los participantes, o que las mejorías en la evocación de recuerdos estuvieran correlacionadas con dichos cambios en la coherencia. Sus resultados sembraron dudas sobre la hipótesis de interacción interhemisférica y pueden tener implicaciones importantes para la investigación sobre el mecanismo neurobiológico subyacente al EMDR.

\section{Monitorización electroencefalográfica en EMDR}

Sin embargo, hasta la fecha, ninguno de estos estudios ha tenido éxito a la hora de investigar con una resolu- ción temporal exacta los disparos en tiempo real de las neuronas cerebrales en respuesta a los estímulos externos inducidos por el EMDR, porque los efectos de la terapia sobre la activación/desactivación cerebral tan sólo fueron registrados antes y después del tratamiento. Esto ha restringido la información presentada a condiciones estáticas que no describen las dinámicas detalladas de la sincronización neuronal regional durante las sesiones de EMDR - un paso esencial en la compresión del mecanismo subyacente a la terapia EMDR. Una de las herramientas que potencialmente puede ayudar a superar los factores metodológicos limitantes es el EEG porque tiene una resolución temporal de milisegundos además de una capacidad aceptable para identificar las fuentes de actividad dentro del espacio tridimensional del cerebro.

$\mathrm{Al}$ investigar por medio del EEG la respuesta funcional antes, durante y después de la terapia EMDR, Pagani et al. (2012) han abierto recientemente nuevos caminos en la divulgación de modificaciones neurofisiopatológicas en tiempo real relacionadas con una sesión de tratamiento de EMDR, para lo cual se había publicado previamente (Pagani et al., 2011) un informe preliminar que describía su metodología y viabilidad. Para permitir que el experimento fuese lo más ecológico y cálido posible para los pacientes, tanto el tratamiento como las grabaciones de EEG tuvieron lugar en una tranquila consulta de un terapeuta privado. Los participantes eran 10 individuos con trauma psíquico importante que habían sido derivados de manera consecutiva a cuatro terapeutas con amplia experiencia en EMDR y 10 controles no traumatizados sanos que accedieron a participar en el estudio. Los terapeutas, todos distinguidos miembros de la Asociación EMDR Italia, se ofrecieron como voluntarios para participar en el estudio $\mathrm{y}$ asegurar que la terapia tuviese el mejor resultado posible.

Se hizo una comparación entre la activación del cerebro en la fase aguda y en la fase de post-tratamiento en las personas traumatizadas durante las sesiones de EMDR, tanto las de guión autobiográfico como las de "modo en vivo". Tales cambios neurobiológicos también se compararon con los que se produjeron en los participantes sanos, que fueron investigados bajo las mismas condiciones experimentales por dos razones: (a) para descartar la posibilidad de que cualquier activación encontrada en los pacientes derivase de los movimientos oculares per se y/o de la simple evocación de un recuerdo autobiográfico y (b) para investigar las posibles diferencias en la activación neuronal entre individuos traumatizados y no traumatizados sometidos a un mismo estímulo psicológico. 
Pagani et al. (2012) señalaron diferencias significativas entre el EEG de pacientes y controles no sintomáticos, tanto al escuchar el guión como durante la estimulación ocular bilateral. Detectaron en los primeros una actividad significativamente mayor en la corteza orbitofrontal, sobre todo en el hemisferio izquierdo. Por el contrario, los controles no traumatizados mostraron en ambos casos una mayor activación en grandes áreas de los lóbulos frontal, temporal y parietal, especialmente en el hemisferio derecho (ver Figura 1).

La comparación entre el EEG de los pacientes durante el EMDR en la primera sesión y la última mostró una activación significativamente mayor durante la última en el córtex temporo-occipital, principalmente en el lado izquierdo (Pagani et al., 2012). En los pacientes después de la terapia, se observó también una disminución significativa de los componentes rápidos de alfa y gamma de la activación presente en la corteza fronto-parietal en la primera sesión de EMDR (véase la Figura 2).

\section{Debate general}

El objetivo principal a la hora de analizar el conjunto de estudios anatómico-funcionales realizados en los últimos 13 años ha sido ampliar nuestro conocimiento sobre la base neurobiológica que subyace al éxito de la terapia EMDR. Esto se ha llevado a cabo utilizando diversas metodologías (neuropsicología, SPECT, MRI y EEG) tratando de divulgar los cambios neuronales en EMDR que ocurren dentro de los diferentes aspectos de la fisiopatología humana, es decir, la neuropsicología, la perfusión sanguínea, la densidad neuronal y la activación eléctrica. Este emocionante viaje ha llevado a confirmar la escasa evidencia inicial de la asociación entre el resultado clínico de la terapia EMDR y algunos cambios en las funciones y estructuras cerebrales y también ha confirmado la viabilidad de la monitorización en tiempo real de las activaciones corticales durante la desensibilización inducida por movimientos oculares bilaterales durante la terapia EMDR. Este éxito técnico y científico también nos ha permitido representar por vez primera las activaciones cerebrales dominantes causadas por las acciones terapéuticas mediadas por un protocolo de terapia de exposición. Esto ha hecho de EMDR la primera psicoterapia con un efecto neurobiológico probado.

En la mayoría de los estudios analizados, durante las fases de escuchar el script se encontró una activación cortical concordante en las áreas límbicas en la etapa en la cual los efectos del trauma son clínicamente evidentes. La activación predominante de estas regiones en la fase sintomática puede explicarse como una hiperactivación emocional durante la re-experimentación del trauma y se considera que representa el correlato neurobiológico de la valencia afectiva del estímulo (Steele y Lawrie, 2004). El estudio de Pagani et al. (2012) también ha confirmado la misma tendencia durante la fase de estimulación ocular bilateral de la terapia EMDR inicial.

La importante normalización de estas activaciones después del procesamiento del trauma puede ser interpretada como el correlato neurológico de la recuperación clínica. Esto habla en favor de un cambio de la atención emotiva hacia las regiones corticales con un rol dominante cognitivo y sensorial. Por lo tanto la retención del recuerdo del evento traumático puede cambiar de un estado subcortical implícito a un estado cortical explícito donde diferentes regiones participan en el procesamiento de la experiencia. Es probable que esto también esté relacionado con un efecto señalado anteriormente del EMDR sobre la memoria a corto plazo durante cuyo procesamiento se activan principalmente áreas asociativas (van den Hout et al., 2011).

Comparando críticamente los resultados del reciente estudio EEG (Pagani et al., 2012) con investigación previa con SPECT y TCC (Lansing et al., 2005; Pagani et al., 2007) se encontró que las mismas regiones cerebrales están implicadas en la respuesta a la terapia EMDR (Figura 3). Esto valida de forma cruzada las dos metodologías e implica que la terapia EMDR está asociada con cambios funcionales en las mismas regiones topográficas que las activadas por el guión autobiográfico (es decir, circunvoluciones fusiformes y corteza occipital y frontal), lo cual confirma su potencia a través de diferentes estudios y grupos completamente diferentes de pacientes y eventos traumáticos.

\section{Conclusiones}

En conclusión, durante la última década, los estudios funcionales y anatómicos han producido resultados muy prometedores que apoyan la evidencia de los modelos neurobiológicos que explican los cambios después de la terapia EMDR y que describen las activaciones corticales específicas para la escucha de script y la desensibilización ocular bilateral. Estos resultados estimulan la continuación de los esfuerzos para desentrañar los mecanismos patofisiológicos subyacentes a este tratamiento altamente eficaz para el TEPT. 

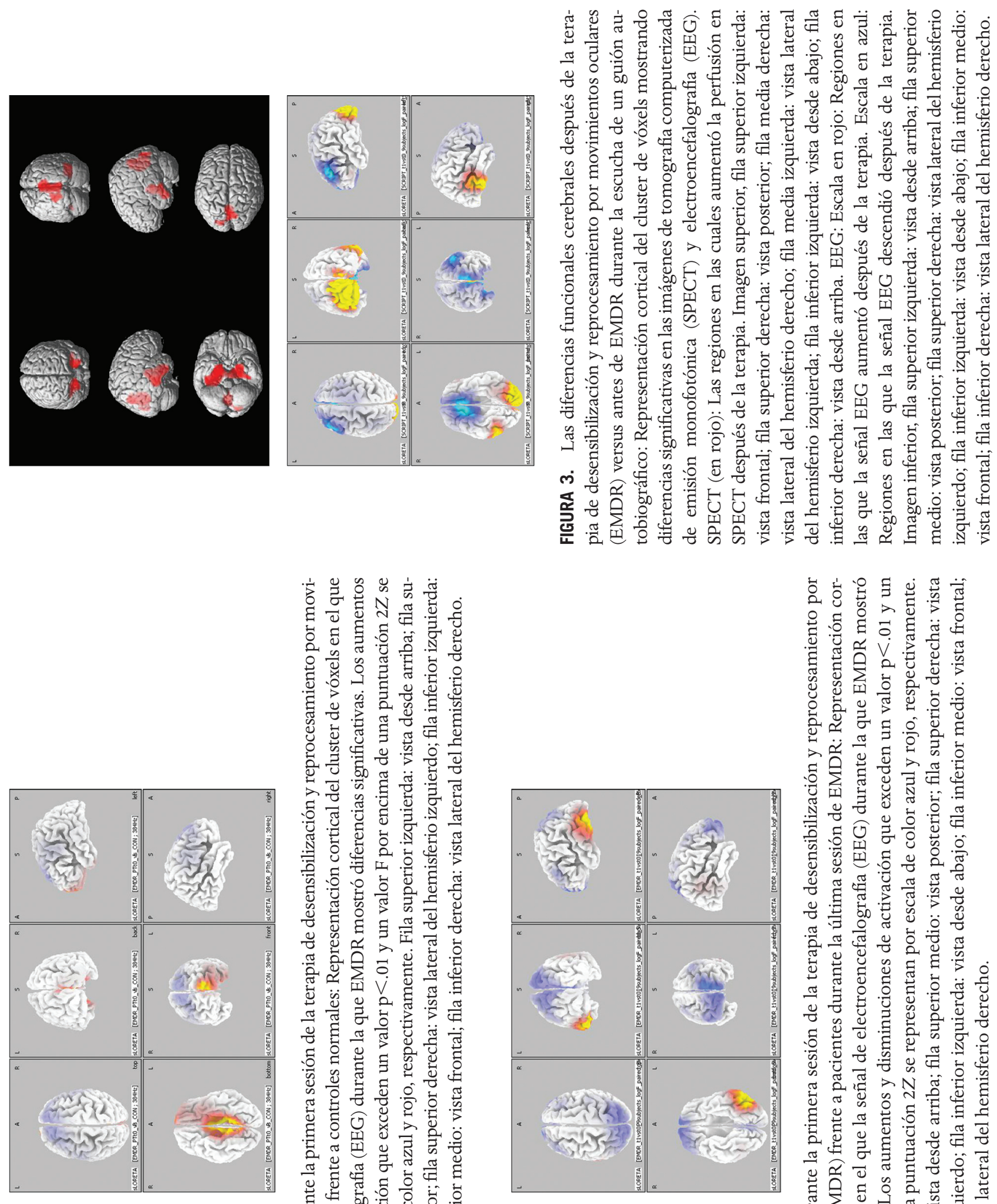

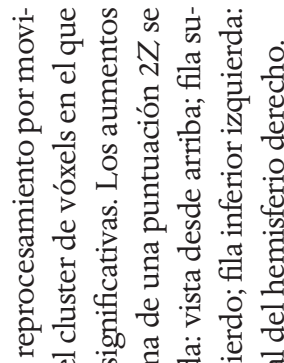

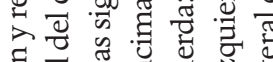

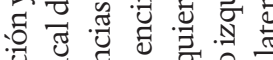

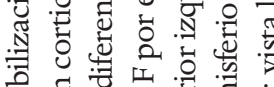

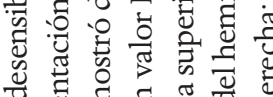

ष

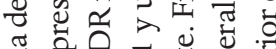

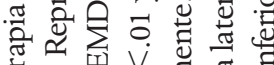

एँ仓

ส

ष $\pi$ ॠ

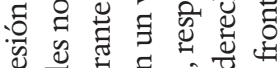

के

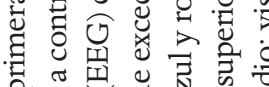

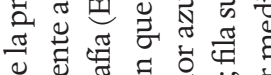

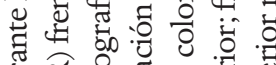

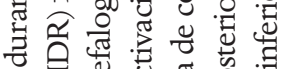

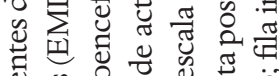

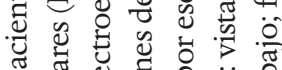

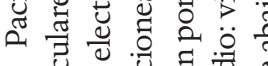

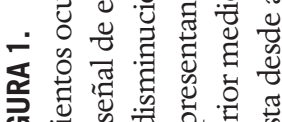

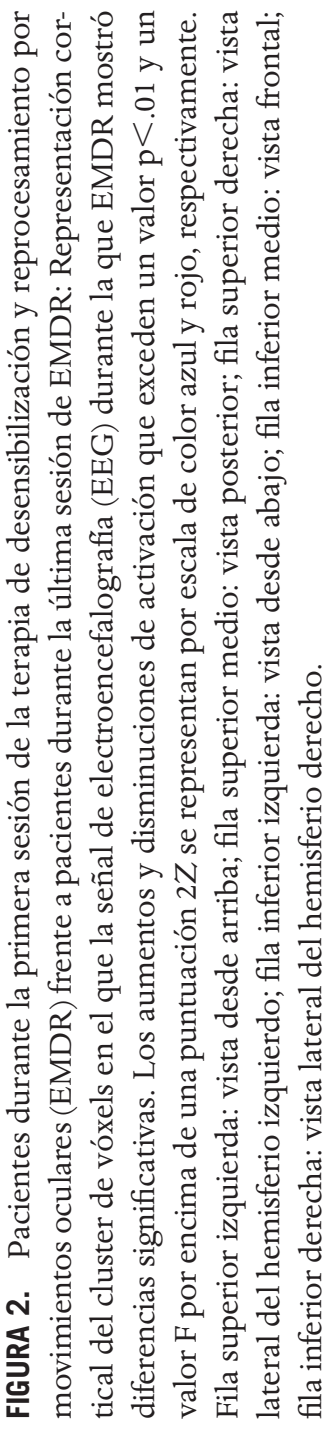




\section{Referencias}

Bender, V. A., Bender, K. J., Brasier, D. J., \& Feldman, D. E. (2006). Two coincidence detectors for spike timing in somatosensory cortex. Journal of Neuroscience, 26, 4166-4177.

Bossini, L., Casolaro, I., Santarnecchi, E., Caterini, C., Koukouna, D., Fernandez, I., \& Fagiolini, A. (2012). Evaluation study of clinical and neurobiological efficacy of EMDR in patients suffering from post-traumatic stress disorder. Rivista di Psichiatria, 47(2 Suppl.), 12-15.

Bradley, R., Green, J., Russ, E., Dutral, L., \& Westen, D. (2005). A multidimensional meta-analysis of psychotherapy for PTSD. American Journal of Psychiatry, 162, 214-227.

Bremner, J. D. (2007). Functional neuroimaging in posttraumatic stress disorder. Expert Review of Neurotherapeutics, 7, 393-405.

Cabeza, R., \& Nyberg, L. (2000). Imaging cognition II: An empirical review of 275 PET and fMRI studies. Journal of Cognitive Neuroscience, 12(1), 1-47.

Davidson, P. R., \& Parker, K. C. H. (2001). Eye movement desensitization and reprocessing (EMDR): A meta-analysis. Journal of Consultant Clinical Psychology, 69, 305-316.

Ehlers, A., Bisson, J., Clark, D. M., Creamer, M., Pilling, S., Richards, D., . . . Yule, W. (2010). Do all psychological treatments really work the same in posttraumatic stress disorder? Clinical Psychology Review, 30, 269-276.

First, M. B., Gibbon, M., Spitzer, R. L., \& Williams, J. B. W. (1997). Structured Clinical Interview for DSM-IV Axis I Disorders (SCID-I). Clinician booklet. Washington, DC: American Psychiatric Association.

Francati, V., Vermetten, E., \& Bremner, J. D. (2007). Functional neuroimaging studies in posttraumatic stress disorder: Review of current methods and findings. Depression and Anxiety, 24, 202-218.

Harper, M. L., Rasolkhani-Kaòhorn, T., \& Drozd, J. F. (2009). On theneural basis of EMDR therapy: Insights from qEEG studies. Traumatology, 15, 81-95.

Högberg, G.,Pagani, M.,Sundin, O., Soares, J.,Aberg-Wistedt, A., Tärnell, B., \& Hällström, T. (2007). On treatment with eye movement desensitization and reprocessing of chronic post-traumatic stress disorder in public transportation workers-a randomized controlled trial. Nordic Journal of Psychiatry, 61, 54-61.

Högberg, G., Pagani, M., Sundin, O., Soares, J., AbergWistedt, A., Tärnell, B., \& Hällström, T. (2008). Treatment of post-traumatic stress disorder with eye movement desensitization and reprocessing: Outcome is stable in 35-month follow-up. Psychiatry Research, 59, 101-108.

Hölscher, C., Anwyl, R., \& Rowan, M. J. (1997). Stimulation on rhythm longdepotentiated by stimulation on the negative phase in area CA1 in vivo. Journal of Neuroscience, 17, 6470-6477.

Lamprecht, F., Köhnke, C., Lempa, W., Sack, M., Matzke, M., \& Münte, T. F. (2004). Event-related potentials and EMDR treatment of post-traumatic stress disorder. Neuroscience Research, 49, 267-272.
Lansing, K., Amen, D. G., Hanks, C., \& Rudy, L. (2005). High-resolution brain SPECT imaging and eye movement desensitization and reprocessing in police officers with PTSD. Journal of Neuropsychiatry and Clinical Neuroscience, 17, 526-532.

Letizia, B., Andrea, F., \& Paolo, C. (2007). Neuroanatomical changes after eye movement desensitization and reprocessing (EMDR) treatment in posttraumatic stress disorder. Journal of Neuropsychiatry and Clinical Neurosciences, 19, 475-476.

Levin, P., Lazrove, S., \& van der Kolk, B. (1999). What psychological testing and neuroimaging tell us about the treatment of posttraumatic stress disorder by eye movement desensitization and reprocessing. Journal of Anxiety Disorders, 13, 159-172.

Lin, C. H., Yeh, S. H., Lu, H. Y., \& Gean, P. W. (2003). The similarities and diversities of signal pathways leading to consolidation of conditioning and consolidation of extinction of fear memory. Journal of Neuroscience, 23, 8310-8317.

Lindauer, R. J., Booij, J., Habraken, J. B., van Meijel, E. P., Uylings, H. B., Olff, M., . . Gersons, B. P. (2008). Effects of psychotherapy on regional cerebral blood flow during trauma imagery in patients with post-traumatic stress disorder: A randomized clinical trial. Psychological Medicine, 38, 543-554.

Lindauer, R. J., Vlieger, E. J., Jalink, M., Olff, M., Carlier, I. V., Majoie C. B., . . . Gersons, B. P. (2005). Effects of psychotherapy on hippocampal volume in out-patients with post-traumatic stress disorder: A MRI investigation. Psychological Medicine, 35, 1421-1431.

Looi, J. C., Lindberg, O., Liberg, B., Tatham, V., Kumar, R., Maller, J., . . . Wahlund, L. O. (2008). Volumetrics of the caudate nucleus: Reliability and validity of a new manual tracing protocol. Psychiatry Research, 163, 279-288.

Looi, J. C., Maller, J. J., Pagani, M., Högberg, G., Lindberg, O., Liberg, B., . . Wahlund, L. O. (2009). Caudate volumes in public transportation workers exposed to trauma in the Stockholm train system. Psychiatry Research, 171, 138-143. Mazard, A., Mazoyer, B., Etard, O., TzourioMazoyer, N.,

Kosslyn, S. M., \& Mellet, E. (2002). Impact of fMRI acoustic noise on the functional anatomy of visual mental imagery. Journal of Cognitive Neuroscience, 14, 172-186.

Nardo, D., Högberg, G., Flumeri, F., Jacobsson, H., Larsson, S. A., Hällström, T., \& Pagani, M. (2011). Selfrating scales assessing subjective well-being and distress correlate with $\mathrm{rCBF}$ in PTSD-sensitive regions. Psychological Medicine, 41, 2549-2561.

Nardo, D., Högberg, G., Looi, J., Larsson, S. A., Hällström, T., \& Pagani, M. (2010). Grey matter changes in posterior cingulate and limbic cortex in PTSD are associated with trauma load and EMDR outcome. Journal of Psychiatry Research, 44, 477-485.

Oh, D. H., \& Choi, J. (2007). Changes in the cerebral perfusion after EMDR. Journal of EMDR Practice and Research, $1,24-30$. 
Ohtani, T., Matsuo, K., Kasai, K., Kato, T., \& Kato, N. (2009). Hemodynamic responses of eye movement desensitization and reprocessing in posttraumatic stress disorder. Neuroscience Research, 65, 375-383.

Pagani, M., Di Lorenzo, G., Verardo, A. R., Nicolais, G., Monaco, L., Lauretti, G., . . . Fernandez, I. (2011). Preintraand post-treatment EEG imaging of EMDRMethodology and preliminary results from a single case. Journal of EMDR Practice and Research, 5, 42-56.

Pagani, M., Di Lorenzo, G., Verardo, A. R., Nicolais, G., Monaco, L., Lauretti, G., . . Fernandez, I. (2012). Neurobiology of EMDR-EEG imaging of treatment efficacy. Plos One, 7, e45753.

Pagani, M., Högberg, G., Salmaso, D., Nardo, D., Tärnell, B., Jonsson, C., . . . Sundin, Ö. (2007). Effects of EMDR psychotherapy on $99 \mathrm{mTc}$-HMPAO distribution in occupation-related post-traumatic stress disorder. Nuclear Medicine Communications, 28, 757-765.

Pagani, M., Högberg, G., Salmaso, D., Tärnell, B., SanchezCrespo, A., Soares, J., . . . Sundin, O. (2005). Regional cerebral blood flow during auditory recall in 47 subjects exposed to assaultive and non-assaultive trauma and developing or not posttraumatic stress disorder. European Archives of Psychiatry and Clinical Neuroscience, 255, 359-365.

Peres, J. F., Newberg, A. B., Mercante, J. P., Simão, M., Albuquerque, V. E., Peres, M. J., \& Nasello, A. G. (2007). Cerebral blood flow changes during retrieval of traumatic memories before and after psychotherapy: A SPECT study. Psychological Medicine, 37, 1481-1491.

Plailly, J., Demarquay, G., \& Royet, J. P. (2009). Cerebral imaging and olfactory disorders: A review. B-ENT, (5 Suppl. 13), 61-69.

Propper, R. E., \& Christman, S. D. (2008). Interhemispheric interaction and saccadic horizontal eye movements: Implications for episodic memory, EMDR, and PTSD. Journal of EMDR Practice and Research, 2(4), 269-281.

Propper, R. E., Pierce, J., Geisler, M. W., Christman, S. D., \& Bellorado, N. (2007). Effect of bilateral eye movements on frontal interhemispheric gamma EEG coherence: Implications for EMDR therapy. Journal of Nervous Mental Disorders, 195, 785-788.

Richardson, P., Williams, S. R., Hepenstall, S., Gregory, L., McKie, S., \& Corrigan, F. (2009). EMDR treatment of a patient with posttraumatic stress disorder. Journal of EMDR Practice and Research, 3, 10-23.

Roffman, J. L., Marci, C. D., Glick, D. M., Dougherty, D. D., $\&$ Rauch, S. L. (2005). Neuroimaging and the functional neuroanatomy of psychotherapy. Psychological Medicine, 35, 1385-1398.

Samara, Z., Elzinga, B. M., Slagter, H. A., \& Nieuwenhuis, S. (2011). Do horizontal saccadic eye movements increase interhemispheric coherence? Investigation of a hypothesized neural mechanism underlying EMDR. Frontiers in Psychiatry, 2, 4.

Shapiro, F. (2001). Eye movement desensitization and reprocessing: Basic Principles, protocols, and procedure (2nd ed.). New York, NY: Guilford Press.

Steele, J. D., \& Lawrie, S. M. (2004). Segregation of cognitive and emotional function in the prefrontal cortex: A stereotactic meta-analysis. Neuroimage, 21, 868-875.

Van den Hout, M. A., Engelhard, I. M., Rijkeboer, M. M., Koekebakker, J., Hornsveld, H., Leer, A., . . . Akse, N. (2011). EMDR: Eye movements superior to beeps in taxing working memory and reducing vividness of recollections. Behavioral Research and Therapy, 49, 92-98.

Van Etten, M. L., \& Taylor, S. (1998). Comparative efficacy of treatments for post-traumatic stress disorder: A metaanalysis. Clinical Psychology and Psychotherapy, 5, 126-144.

Agradecimientos. Los autores desean agradecer a Dª Emanuela Enrico por su ayuda en la edición del inglés.

La correspondencia relacionada con este artículo debe dirigirse a Marco Pagani, Instituto de ciencias y tecnologías cognitivas, CNR, Via Palestro 32, 00185, Roma, Italia. E-mail: marco.pagani@istc.cnr.it 